\title{
Interaction (Election Campaigning Communication)
}

\section{AUTHOR}

Desiree Steppat, Laia Castro Herrero

\section{KEYWORDS}

election campaigns, interaction, campaign strategies, interactivity, discussion, engagement

\section{BRIEF DESCRIPTION}

Interaction is described as a way to persuade citizens through direct contact allowing for a dialogical encounter between political actors and citizens (Magin, Podschuweit, Haßler, \& Russmann, 2017). Although the new online environment can facilitate direct communication between politicians and citizens, empirical findings indicate that, to date, a unidirectional communication style between voters and politicians predominates (Jackson \& Lilleker, 2010; Lilleker \& Koc-Michalska, 2013; Stromer-Galley, 2000). To a large extent, politicians still employ the broadcasting style for campaign communication (Graham, Broersma, Hazelhoff, \& van ,t Haar, 2013) and retain communication strategies from the mass media era (Margolis \& Resnick, 2000), as few voters visit their websites on a regular basis (Gibson \& McAllister, 2011) or follow politicians' profiles on social media (Vaccari \& Nielsen, 2013). However, research in campaign communication also shows that the Web 2.0 provide new opportunities for politicians to address an expanded, new electorate and engage them. As an example, studies show that posts that are frequently liked, commented, or shared can reach a much wider circle of users known as secondary audience or second-degree followers (Jacobs \& Spierings, 2016; Vaccari \& Valeriani, 2015). Interaction through social media channels furthermore enables face-to-face-like communication with individual voters, with whom politicians can also ex- change ideas and negotiate campaign strategies (Magin et al., 2017).

FIELD OF APPLICATION/THEORETICAL FOUNDATION In recent years, interaction has been recognized as a central aspect of dialogical communication in the field of public relations (Sweetser \& Lariscy, 2008; Taylor \& Kent, 2004). The theory states that symmetrical and dialogical two-way communication between an organization and its audience can sustainably support relationship building and their maintenance (Zhang \& Seltzer, 2010). By applying this approach to the field of online political communication, it is possible to understand the interactions between politicians and citizens as a form of strategic communication and how they attract and persuade voters.

\section{REFERENCES/COMBINATION WITH OTHER}

\section{METHODS OF DATA COLLECTION}

Interaction in the last twenty years has been mostly studied in the context of the online environment either by looking at structural features of candidates' online tools that enable interactions with users (e.g., Druckman, Kifer, \& Parkin, 2007, 2009; Schweitzer, 2008); or by studying actual interactions between candidates and citizens on social media (e.g., Graham et al., 2013; Klinger, 2013). Both quantitative manual and automated content analyses thereof have been employed to in research on social media interactions). Quantitative content analysis have been also been combined and compared with qualitative interviews with campaign managers (e.g., Magin et al., 2017).

\section{EXAMPLE STUDIES}

see Table 1 


\section{REFERENCES}

Bene, M. (2017). Go Viral on the Facebook! Interactions between Candidates and Followers on Facebook during the Hungarian General Election Campaign of 2014. Information, Communication \& Society, 20(4), 513-529. https://doi.or g/10.1080/1369118X.2016.1198411

Druckman, J. N., Kifer, M. J., \& Parkin, M. (2007). The Technological Development of Congressional Candidate Web Sites. Social Science Computer Review, 25(4), 425-442. https://doi.org/10.1177/0894439307305623

Druckman, J. N., Kifer, M. J., \& Parkin, M. (2009). Campaign Communications in U.S. Congressional Elections. American Political Science Review, 103(3), 343-366. https:// doi.org/10.1017/S0003055409990037

Gibson, R. K., \& McAllister, I. (2011). Do online election campaigns win votes? The 2007 Australian "YouTube" election. Political Communication, 28(2), 227-244.

Graham, T., Broersma, M., Hazelhoff, K., \& van ,t Haar, G. (2013). Between Broadcasting Political Messages and Interacting with Voters. Information, Communication \& Society, 16(5), 692-716. https:// doi.org/10.1080/1369118X.2013.785581

Graham, T., Jackson, D., \& Broersma, M. (2016). New Platform, Old Habits? Candidates' Use of Twitter during the 2010 British and Dutch General Election Campaigns. New Media \& Society, 18(5), 765-783. https:// doi.org/10.1177/1461444814546728

Jackson, N., \& Lilleker, D. G. (2010). Tentative Steps towards Interaction. Internet Research, 20(5), 527-544. https:// doi.org/10.1108/10662241011084103

Jacobs, K., \& Spierings, N. (2016). Social Media, Parties, and Political Inequalities. New York: Palgrave Macmillan US. https://doi.org/10.1057/9781137533906

Keller, T. R., \& Kleinen-von Königslöw, K. (2018). Pseudo-Discursive, Mobilizing, Emotional, and Entertaining: Identifying Four Successful Communication Styles of Political Actors on Social Media during the 2015 Swiss National Elections. Journal of Information Technology \& Politics, 15(4), 358-377. https:// doi.org/10.1080/19331681.2018.1510355

Klinger, U. (2013). Mastering the Art of Social Media. Information, Communica- tion \& Society, 16(5), 717-736. https://

doi.org/10.1080/1369118X.2013.782329

Lilleker, D. G., \& Koc-Michalska, K. (2013). Online Political Communication Strategies: MEPs, E-Representation, and Self-Representation. Journal of Information Technology \& Politics, 10(2), 190-207. https:// doi.org/10.1080/19331681.2012.758071

Lukamto, W., \& Carson, A. (2016). Politweets: Social Media as a Platform for Political Engagement between Victorian Politicians and Citizens. Communication Research and Practice, 2(2), 191-212. https:// doi.org/10.1080/22041451.2016.1186485

Magin, M., Podschuweit, N., Haßler, J., \& Russmann, U. (2017). Campaigning in the Fourth Age of Political Communication. A Multi-Method Study on the Use of Facebook by German and Austrian Parties in the 2013 National Election Campaigns. Information, Communication \& Society, 20(11), 1698-1719. https://doi. org/10.1080/1369118X.2016.1254269

Margolis, M., \& Resnick, D. (2000). Politics as Usual: The Cyberspace „Revolution". Contemporary American politics. Thousand Oaks: Sage.

Schweitzer, E. J. (2008). Innovation or Normalization in E-Campaigning? European Journal of Communication, 23(4), 449-470. https://doi.org/10.1177/0267323108096994

Stromer-Galley, J. (2000). On-line Interaction and Why Candidates Avoid It. Journal of Communication, 50(4), 111-132.

Sweetser, K. D., \& Lariscy, R. W. (2008). Candidates make good friends: An analysis of candidates' uses of Facebook. International Journal of Strategic Communication, 2(3), 175-198.

Taylor, M., \& Kent, M. L. (2004). Congressional web sites and their potential for public dialogue. Atlantic Journal of Communication, 12(2), 59-76.

Vaccari, C., \& Nielsen, R. K. (2013). What drives politicians' online popularity? An analysis of the 2010 US midterm elections. Journal of Information Technology \& Politics, 10(2), 208-222.

Vaccari, C., \& Valeriani, A. (2015). Follow the leader! Direct and indirect flows of political communication during the 2013 Italian general election campaign. New 
Vaccari, C., \& Valeriani, A. (2015). Follow the leader! Direct and indirect flows of political communication during the 2013 Italian general election campaign. New Media \& Society, 17(7), 1025-1042. https:// doi.org/10.1177/1461444813511038

Zhang, W., \& Seltzer, T. (2010). Another piece of the puzzle: Advancing social capital theory by examining the effect of political party relationship quality on political and civic participation. International Journal of Strategic Communication, 4(3), 155-170

Table 1. Overview exemplary studies measuring interaction, discussion, participation, and related constructs.

\begin{tabular}{|c|c|c|c|c|}
\hline Study & Medium & Constructs & Operationalization & Coding \\
\hline $\begin{array}{l}\text { Druckman } \\
\text { et al. (2007); } \\
\text { Druckman et } \\
\text { al. (2009) }\end{array}$ & $\begin{array}{l}\text { Candidate } \\
\text { websites }\end{array}$ & Interactivity & $\begin{array}{l}\text { Web sites were scrutinized in light } \\
\text { of their ability to create someform } \\
\text { of interaction by e.g.enabling users } \\
\text { to personalize information, arrange } \\
\text { information, add information, and/ } \\
\text { or communicate with other voters } \\
\text { and/or the candidate }\end{array}$ & Additive index \\
\hline $\begin{array}{l}\text { Schweitzer } \\
\text { (2008) }\end{array}$ & $\begin{array}{l}\text { Candidate } \\
\text { websites }\end{array}$ & $\begin{array}{l}\text { Interactivity } \\
\text { provision } \\
\text { strategies }\end{array}$ & $\begin{array}{l}\text { Possibility to comment on news; } \\
\text { Agenda can be updated by visitors; } \\
\text { A channel on video sharing web- } \\
\text { sites; Possibility to comment (a } \\
\text { video sharing website); Life web- } \\
\text { cam; Online photo gallery; Possi- } \\
\text { bility to comment (online photo } \\
\text { gallery); Easy contact; Online polls; } \\
\text { Profile on SNS; Online forum or } \\
\text { chat (among visitors); Online forum } \\
\text { or chat (with politicians); Possibility } \\
\text { to share content of the website; Pos- } \\
\text { sibility to share content on social } \\
\text { media; Information about political } \\
\text { program (interactive format) }\end{array}$ & Additive index \\
\hline $\begin{array}{l}\text { Magin et al. } \\
\text { (2017) }\end{array}$ & $\begin{array}{l}\text { Facebook } \\
\text { posts }\end{array}$ & Interaction & $\begin{array}{l}\text { Index including (1) number of par- } \\
\text { ties' comments, ( } 2 \text { ) the number of } \\
\text { users' comments per } 1,000,000 \text { eligi- } \\
\text { ble voters, and (3) the share of posts } \\
\text { in which the parties encourage the } \\
\text { voters to discuss politics on the par- } \\
\text { ties' Facebook page (reciprocity). }\end{array}$ & $\begin{array}{l}\text { Combined } \\
\text { index }\end{array}$ \\
\hline
\end{tabular}




\begin{tabular}{|c|c|c|c|c|}
\hline Study & Medium & Constructs & Operationalization & Coding \\
\hline $\begin{array}{l}\text { Graham et } \\
\text { al. (2013), } \\
\text { Graham, } \\
\text { Jackson, and } \\
\text { Broersma } \\
(2016)\end{array}$ & $\begin{array}{l}\text { Twitter } \\
\text { posts }\end{array}$ & Interaction & $\begin{array}{l}\text { Tweets including: Debating/position } \\
\text { taking; Acknowledging; Organizing/ } \\
\text { mobilizing; Advice giving/helping; } \\
\text { and/or Consulting } \\
\text { Furthermore @Tweets were scruti- } \\
\text { nized with whom politicians inter- } \\
\text { acted: Public; Politician/candidate; } \\
\text { journalist/media; Party activist; } \\
\text { Lobbyist; Expert; Celebrity; Indus- } \\
\text { try; and/or Authority }\end{array}$ & $\begin{array}{l}\text { (0) Not pre- } \\
\text { sent } \\
\text { (1) Present }\end{array}$ \\
\hline $\begin{array}{l}\text { Lukamto } \\
\text { and Carson } \\
(2016)\end{array}$ & $\begin{array}{l}\text { Twitter } \\
\text { com- } \\
\text { ments, @ } \\
\text { menti- } \\
\text { ons, and } \\
\text { retweets } \\
\text { (RTs) }\end{array}$ & Discussion & $\begin{array}{l}\text { Measures quantity of one-way } \\
\text { and two-way messages between } \\
\text { members of parliament (MPs) and } \\
\text { citizens and who they interact with: } \\
\text { 'citizen to politician'; 'politician to } \\
\text { citizen'; or 'politician to politician' }\end{array}$ & Count variable \\
\hline Bene (2017) & $\begin{array}{l}\text { Facebook } \\
\text { posts }\end{array}$ & Engagement & $\begin{array}{l}\text { Engagement content is coded if the } \\
\text { post contains either requests for } \\
\text { likes, comments, and/or sharing or } \\
\text { whether it } \\
\text { poses a question. All of these indi- } \\
\text { vidual elements were also coded on } \\
\text { their own and analyzed in specified } \\
\text { models with all dependent variables }\end{array}$ & $\begin{array}{l}\text { (0) Not present } \\
\text { (1) Present }\end{array}$ \\
\hline $\begin{array}{l}\text { Klinger } \\
(2013)\end{array}$ & $\begin{array}{l}\text { Facebook } \\
\text { \& Twitter } \\
\text { posts }\end{array}$ & $\begin{array}{l}\text { Participa- } \\
\text { tion }\end{array}$ & $\begin{array}{l}\text { Posts including calls for discussion, } \\
\text { appeals to collect signatures and } \\
\text { mobilize other people to participate } \\
\text { and to vote as well as general com- } \\
\text { munity-building }\end{array}$ & $\begin{array}{l}\text { (0) Not present } \\
\text { (1) Present }\end{array}$ \\
\hline $\begin{array}{l}\text { Keller and } \\
\text { Kleinen-von } \\
\text { Königslöw } \\
(2018)\end{array}$ & $\begin{array}{l}\text { Facebook } \\
\text { \& Twitter } \\
\text { posts }\end{array}$ & $\begin{array}{l}\text { Pseudo } \\
\text { discursive } \\
\text { style }\end{array}$ & & $\begin{array}{l}\text { (0) Not present } \\
\text { (1) Present }\end{array}$ \\
\hline $\begin{array}{l}\text { Graham et } \\
\text { al. (2013), } \\
\text { Graham et } \\
\text { al. (2016) }\end{array}$ & $\begin{array}{l}\text { Twitter } \\
\text { posts }\end{array}$ & Interaction & $\begin{array}{l}\text { Tweets including: Debating/position } \\
\text { taking; Acknowledging; Organizing/ } \\
\text { mobilizing; Advice giving/helping; } \\
\text { and/or Consulting } \\
\text { @Tweets were also scrutinized with } \\
\text { whom politicians interacted: Public; } \\
\text { Politician/candidate; journalist/me- } \\
\text { dia; Party activist; Lobbyist; Expert; } \\
\text { Celebrity; Industry; and/or Authority }\end{array}$ & $\begin{array}{l}\text { (0) Not present } \\
\text { (1) Present }\end{array}$ \\
\hline
\end{tabular}

